\title{
1 THz span optical frequency comb using VCSELs and off the Shelf expansion techniques
}

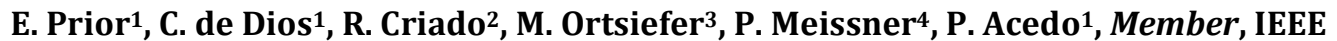 \\ 1. Electronics Technology Department, Universidad Carlos III de Madrid, Leganés, 28911 Spain \\ 2. Luz WaveLabs S.L. Av. Gregorio Peces Barba, Leganés, 28919 Spain \\ 3. Vertilas GmbH. Garching, Germany \\ 4. Technische Universität Darmstadt, Darmstadt, Germany \\ eprior@ing.uc3m.es
}

\begin{abstract}
We show, to our knowledge, the broadest VCSEL-based optical comb with 1THz span $(20 \mathrm{~dB}), 193$ teeth, obtained by cascading two expansion stages, one with Electro-Optic Modulators and the second based on High Non-Linear Fibers.

OCIS codes: 250.7260 Vertical cavity surface emitting lasers; 230.0250 Optoelectronics; 060.4370 Nonlinear optics, fibers;
\end{abstract}

In this work, we present our latest results on VCSEL-based Optical Frequency Comb (OFC) generation and expansion using commercially available components. The result is a versatile, and compact comb architecture, that offers cost and energy efficiency. Our OFCs are based on Vertical-Cavity Surface-Emitting Lasers (VCSELs) under Gain Switching (GS) regime that provide record combs in energy efficiency and mode coherence compared to other laser diodes (LD) technologies [1]-[3]. Among others, these systems are useful in fields of application like spectroscopy, THz generation or green optical communications [4]-[8].

Up to now, the optical frequency span was the main limiting factor when comparing VCSELs with other laser technologies for OFCs, but here we present a breakthrough in this limit: we have obtained the broadest VCSELbased optical comb, of $1 \mathrm{THz}(20 \mathrm{~dB})$ span, which is a record value to our knowledge. This comb has 193teeth in the $20 \mathrm{~dB}$ span which corresponds to $9.1 \mathrm{~nm}$ in wavelength. The distance between comb teeth is $5 \mathrm{GHz}$ and the overall power consumption of the components in the set-up is lower than $10 \mathrm{~W}$. However, with this configuration, the flatness of the comb and its dynamic range still leave room for improvement.

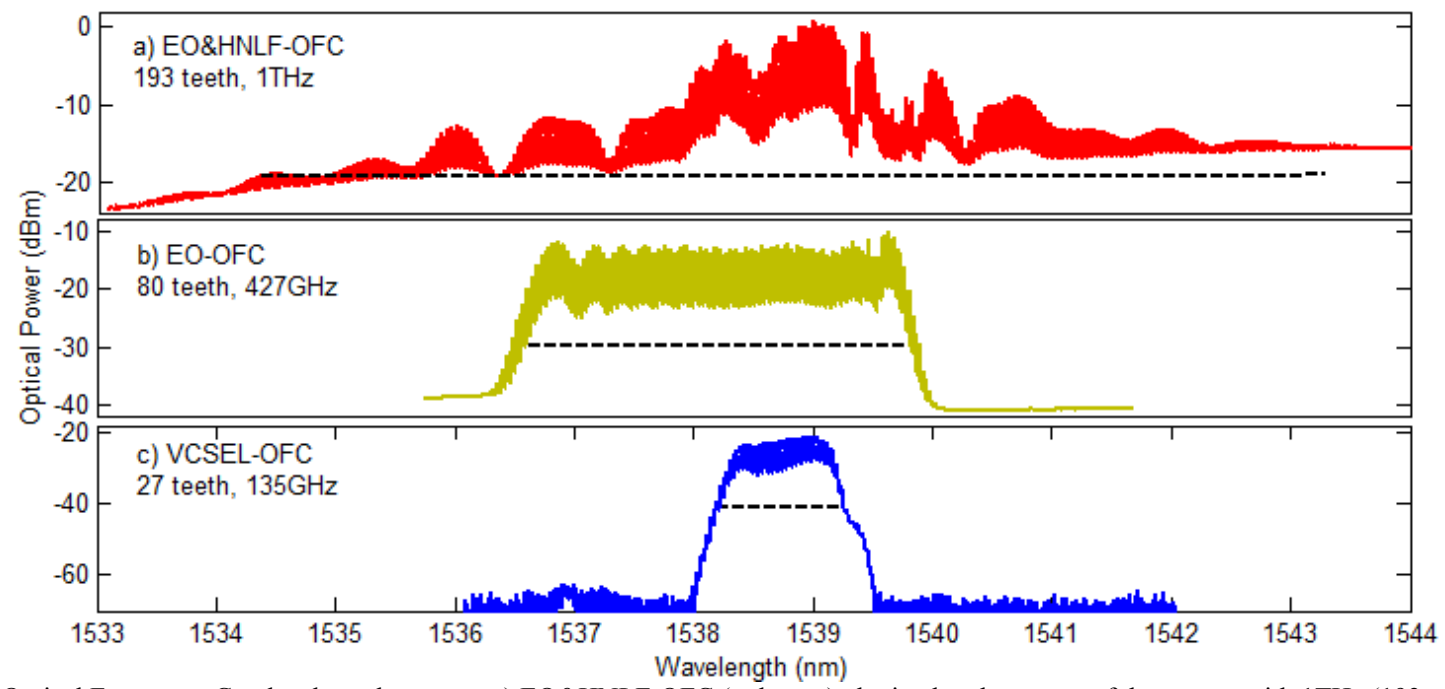

Fig. 1 Optical Frequency Combs along the set-up. a) EO\&HNLF-OFC (red trace) obtained at the output of the set-up, with 1THz (193 teeth) of $20 \mathrm{~dB}$ span. b) EO-OFC (yellow trace) obtained at the output of the first expansion stage based on PMs, with $427 \mathrm{GHz}$ ( 80 teeth) in the $2 \mathrm{~dB}$ span.

c) VCSEL-OFC (blue trace) obtained at the output of the VCSEL under GS regime, with $135 \mathrm{GHz}(27$ teeth) in the span. The VCSEL supply parameters are $20^{\circ} \mathrm{C}, 11.4 \mathrm{~mA}, 5.4 \mathrm{GHz}, 16 \mathrm{dBm}$.

In Fig. 1a, we observe the reported 1THz comb, called EO\&HNLF-OFC (red line) and its 20dB span (black dotted line). The dynamic range of this spectrum is lower than $20 \mathrm{~dB}$ in the upper wavelengths so the $20 \mathrm{~dB}$ line covers the span where the teeth are at least $0.5 \mathrm{~dB}$ above the noise level (the OSA used has $0.002 \mathrm{~nm}$ resolution). In order to obtain this spectrum we have implemented a set-up that combines two different expansion techniques as explained in the following paragraph. 
The optical source starts with a VCSEL set at $20^{\circ} \mathrm{C}$ and $11.4 \mathrm{~mA}$ of bias current. The VCSEL is operating in GS regime using a $16 \mathrm{dBm}$ tone at $5.4 \mathrm{GHz}$. The obtained comb, called VCSEL-OFC (blue trace in Fig. 1c), has 27 teeth in the $20 \mathrm{~dB}$ span that corresponds to $135 \mathrm{GHz}$ (previously presented in [1], [2], [9]). This VCSEL-OFC enters the first expansion stage, with two Electro Optical (EO) Phase Modulators (PM). Cascading several EO modulators is one of the most common techniques for comb generation and expansion [10]. The obtained comb EO-OFC (yellow trace in Fig. 1b), has 80 teeth in the $20 \mathrm{~dB}$ span that corresponds to $427 \mathrm{GHz}$. Finally, the second expansion stage is based on Highly Non-Linear Fiber (HNLF). Before this element, we include a Dispersion Compensating Fiber (DCF) to compensate the dispersion and an Erbium Dopped Fiber Amplifier (EDFA) in order to increase the peak power of the pulse and enhance the non-linear effects in the HNLF. In Fig. 2 the complete set-up is shown, which is similar to the ones described to expand combs with different laser technologies in [10], [11].

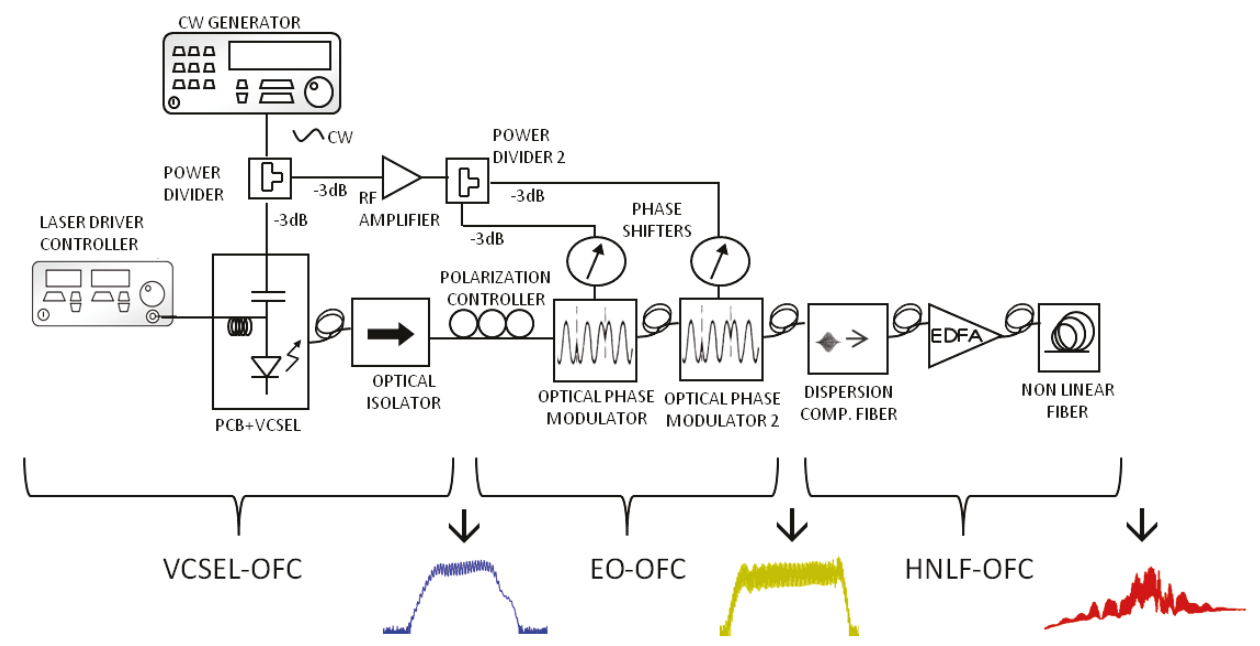

Fig. 2 Set-up used for the EO\&HNLF-OFC. VCSEL-OFC (blue trace) is the output of the VCSEL under GS regime. A first expansion stage called EO-OFC is formed by two PMs and provides an output comb of $427 \mathrm{GHz}$ in the $20 \mathrm{~dB}$ span (yellow trace). Then, the second expansion stage called HNLF-OFC is formed by a DCF, an EDFA and a HNLF and provides an output comb called EO\&HNLF-OFC (red trace), which is the $1 \mathrm{THz}$ broad optical frequency comb. See text for more details.

This is a remarkable result and our efforts continue in order to improve the flatness and the dynamic range of this $1 \mathrm{THz}$ broad comb. We will also continue analyzing the coherence between the teeth in the comb and other features that characterizes a comb spectra and a comb system. At the end we focus on the improvement of the quality and energy efficiency of these VCSEL-based OFCs.

\section{References}

[1] E. Prior, C. de Dios, A. R. Criado, M. Ortsiefer, P. Meissner, and P. Acedo, "Experimental study of VCSEL-based Optical Frequency Comb Generators," IEEE Photonics Technol. Lett., vol. 26, no. 21, pp. 1-1, Nov. 2014.

[2] E. Prior, C. de Dios, M. Ortsiefer, P. Meissner, and P. Acedo, "Dual-polarization VCSEL-based optical frequency comb generation," in The European Conference on Lasers and Electro-Optics, 2015, p. CB_P_11.

[3] E. Prior, C. De Dios, M. Ortsiefer, P. Meissner, and P. Acedo, "Understanding VCSEL-Based Gain Switching Optical Frequency Combs: Experimental Study of Polarization Dynamics,” J. Light. Technol., vol. 33, no. 22, pp. 4572-4579, Nov. 2015.

[4] P. J. Delfyett, I. Ozdur, N. Hoghooghi, M. Akbulut, J. Davila-Rodriguez, and S. Bhooplapur, "Advanced Ultrafast Technologies Based on Optical Frequency Combs,” IEEE J. Sel. Top. Quantum Electron., vol. 18, no. 1, pp. 258-274, Jan. 2012.

[5] C. Chen, C. Zhang, D. Liu, K. Qiu, and S. Liu, "Tunable optical frequency comb enabled scalable and cost-effective multiuser orthogonal frequency-division multiple access passive optical network with source-free optical network units," Optics Letters, vol. 37. p. $3954,2012$.

[6] A. R. Criado, C. de Dios, E. Prior, G. H. Dohler, S. Preu, S. Malzer, H. Lu, A. C. Gossard, and P. Acedo, "Continuous-Wave Sub-THz Photonic Generation With Ultra-Narrow Linewidth, Ultra-High Resolution, Full Frequency Range Coverage and High Long-Term Frequency Stability," IEEE Trans. Terahertz Sci. Technol., vol. 3, no. 4, pp. 461-471, Jul. 2013.

[7] P. Martin-Mateos, M. Ruiz-Llata, J. Posada-Roman, and P. Acedo, "Dual-Comb Architecture for Fast Spectroscopic Measurements and Spectral Characterization," IEEE Photonics Technol. Lett., vol. 27, no. 12, pp. 1309-1312, Jun. 2015.

[8] R. Wu, C. M. Long, D. E. Leaird, and A. M. Weiner, "Directly Generated Gaussian-Shaped Optical Frequency Comb for Microwave Photonic Filtering and Picosecond Pulse Generation," IEEE Photonics Technol. Lett., vol. 24, no. 17, pp. 1484-1486, Sep. 2012.

[9] A. R. Criado, C. de Dios, E. Prior, M. Ortsiefer, P. Meissner, and P. Acedo, "VCSEL-Based Optical Frequency Combs: Toward Efficient Single-Device Comb Generation,” IEEE Photonics Technol. Lett., vol. 25, no. 20, pp. 1981-1984, Oct. 2013.

[10] R. Wu, V. Torres-Company, D. E. Leaird, and A. M. Weiner, "Supercontinuum-based 10-GHz flat-topped optical frequency comb generation," Opt. Express, vol. 21, no. 5, p. 6045, Mar. 2013.

[11] T. Yang, J. Dong, S. Liao, D. Huang, and X. Zhang, "Comparison analysis of optical frequency comb generation with nonlinear effects in highly nonlinear fibers.," Opt. Express, vol. 21, no. 7, pp. 8508-20, Apr. 2013. 